\section{Medical Principles and Practice}

Al-Mutawa, S. 43

Al-Nakib, W. 47

Altawalah, H. 47

Ariga, J. 43

Behbehani, J.M. 1, 10

Behbehani, K. 32
Honkala, E. 1,17

Honkala, S. 24

Mumghamba, E.G. 69

Naidoo, S. 60

Nazar, H. 43
Petersen, P.E. 3

Vernillo, A.T. 60

Williams, D.M. 52

\title{
Subject Index Vol. 23, Suppl. 1, 2014
}

Annual symposia 47

Atraumatic restorative treatment 17

Collaboration 60

Common risk factors 52

Community of practice 60

Dental curriculum 69

- health education 17

- workforce 69

Diabetes 32

Global burden of oral disease 3

- health 60

Health behaviour 24

- ethics 60

- inequalities 52

- policy 17

- promotion 24,52
Healthy living 32

HIV workshop 47

Inequity in oral health 3

Integrated disease prevention 3

Kuwait 32

Lifestyle 32

Muhimbili dental curriculum 69

Non-communicable disease 52

Obesity 32

Oral epidemiology 10

- health 10,43

- - care coverage 3

- _ $\quad$ - systems 69

- - promotion 3,17
Population 32

Prevention 43, 52

Preventive dentistry 10

Primary health care 10,17

- oral health care 69

Public health 60

School 32

- children 24, 43

Social capital 60

- determinants of health 52

- factors 32

WHO collaborating centres 3

- collaboration centre 47 